\title{
Secondary glaucoma in CAPN5-associated neovascular inflammatory vitreoretinopathy
}

\author{
This article was published in the following Dove Press journal: \\ Clinical Ophthalmology \\ 27 June 2016 \\ Number of times this article has been viewed
}

\author{
Abdourahman Cham ${ }^{1,2}$ \\ Mayank Bansal ${ }^{3}$ \\ Himanshu K Banda ${ }^{4}$ \\ Young Kwon' \\ Paul S Tlucek' \\ Alexander G Bassuk ${ }^{5}$ \\ Stephen H Tsang ${ }^{6,7}$ \\ Warren M Sobol $^{8}$ \\ James C Folk' \\ Steven Yeh $^{4}$ \\ Vinit B Mahajan ${ }^{1,2}$ \\ 'Department of Ophthalmology and \\ Visual Sciences, ${ }^{2}$ Omics Laboratory, \\ University of lowa, lowa City, IA, \\ USA; ${ }^{3}$ Rajendra Prasad Centre \\ for Ophthalmic Sciences, All India \\ Institute of Medical Sciences, \\ New Delhi, India; ${ }^{4}$ Department of \\ Ophthalmology, Emory University \\ School of Medicine, Atlanta, GA, \\ ${ }^{5}$ Department of Pediatrics, University \\ of lowa, lowa City, IA, ${ }^{6}$ Barbara and \\ Donald Jonas Laboratory of Stem \\ Cells and Regenerative Medicine and \\ Bernard and Shirlee Brown Glaucoma \\ Laboratory, Department of Pathology \\ and Cell Biology, Institute of Human \\ Nutrition, College of Physicians \\ and Surgeons, Columbia University, \\ ${ }^{7}$ Edward S Harkness Eye Institute, \\ New York-Presbyterian Hospital, \\ New York, NY, ${ }^{8}$ Retina Physicians \& \\ Surgeons, Inc., Dayton, OH, USA
}

Correspondence: Vinit B Mahajan Department of Ophthalmology and Visual Sciences, University of lowa, 200 Hawkins Drive, lowa City, IA 52242, USA

$\mathrm{Tel}+\mid 319467515$ I

Fax + I 3193560363

Email mahajanlab@gmail.com
Objective: The objective of this study was to review the treatment outcomes of patients with secondary glaucoma in cases of autosomal dominant neovascular inflammatory vitreoretinopathy (ADNIV), a hereditary autoimmune uveitis due to mutations in CAPN5.

Patients and methods: A retrospective, observational case series was assembled from ADNIV patients with secondary glaucoma. The main outcome measures were intraocular pressure (IOP), visual acuity, use of antiglaucoma medications, ocular surgeries, and adverse outcomes. Perimetry and optic disk optical coherence tomography (OCT) were also analyzed.

Results: Nine eyes of five ADNIV patients with secondary glaucoma were reviewed. Each received a fluocinolone acetonide (FA) implant for the management of posterior uveitis. Following implantation, no eyes developed neovascular glaucoma. Five eyes (in patients 1, 2, and 5) required Ahmed glaucoma valve surgery for the management of steroid-responsive glaucoma. Patient 2 also developed angle closure with iris bombe and underwent laser peripheral iridotomy. Patient 4 had both hypotony and elevated IOP that required periodic antiglaucoma medication in the FA-implanted eye. Patient 3 did not develop steroid-response glaucoma in either eye. Optic disk examinations were obscured by fibrosis and better assessed with OCT.

Conclusion: ADNIV patients show combined mechanism secondary glaucoma best assessed by OCT of the optic disk. The FA implants have reduced uveitic and neovascular glaucoma. Nevertheless, IOP management remains complex due to steroid-response glaucoma, angle closure glaucoma, and hypotony.

Keywords: ADNIV, CAPN5, calpain-5, fluocinolone acetonide, secondary glaucoma, uveitis

\section{Introduction}

Autosomal dominant neovascular inflammatory vitreoretinopathy (ADNIV) is an inherited autoimmune uveitis and neovascular vitreoretinal degeneration caused by mutations in the CAPN5 gene. ${ }^{1-3}$ CAPN5 is an intracellular protease expressed in the retina. The disease alleles appear to increase CAPN5 proteolytic activity, ${ }^{4}$ but molecular disease mechanisms remain to be elucidated. Inflammatory cells in the vitreous and anterior chamber, photoreceptor degeneration, vitreous hemorrhages, epiretinal membranes (ERMs), and proliferative iris and retinal neovascularization characterize ADNIV. Owing to intraocular fibrosis, there is further progression to tractional retinal detachment and eventual phthisis. ${ }^{1,2}$

ADNIV patients develop glaucoma secondary to uveitis, steroid response, and angle neovascularization. Autoimmune uveitis can lead to elevated intraocular pressure (IOP) and uveitic glaucoma due to obstruction of trabecular drainage by inflammatory infiltrates and proteinaceous materials. ${ }^{5}$ However, anti-inflammatory therapy with steroids can increase IOP and predispose ADNIV patients to steroid-induced glaucoma. 
ADNIV patients are also at risk for neovascular glaucoma. There is rubeotic and neovascular glaucoma in nearly half of ADNIV patients older than 60 years. ${ }^{1}$ Therefore, ADNIV patients have multiple risk factors for developing glaucoma. In late stages, some ADNIV eyes become hypotonous, which could accelerate phthisis. Given their common genetic defect, ADNIV patients provide a unique perspective on the management of uveitic glaucoma.

Management of glaucoma in ADNIV, especially in steroid responders, can be challenging given the competing interests of effective anti-inflammatory therapy and control of elevated IOP. ${ }^{6}$ ADNIV patients often receive fluocinolone acetonide (FA) implants for the management of chronic uveitis. Steroid-sparing agents have limited efficacy in ADNIV uveitis, and the FA implants obviate the need for long-term oral steroid therapy. ${ }^{7}$ Surgical management of uveitic glaucoma with glaucoma drainage devices has been described. ${ }^{8,9}$ In this series, we report on five patients with ADNIV-associated uveitis and describe their treatment outcomes.

\section{Patients and methods}

The study was approved by the Institutional Review Board for Human Subjects Research and adhered to the tenets of the Declaration of Helsinki. All patients provided written informed consent. A retrospective case series was assembled from the charts of ADNIV patients (2001 to present) from the University of Iowa (four charts) and Emory University (one chart). Clinical examinations were performed by vitreoretinal and glaucoma specialists (authors). Genetic testing was performed as previously described. ${ }^{2}$ The FA implantation surgery was performed as previously described. ${ }^{10}$ The Ahmed glaucoma valve (AGV) implantation surgery was performed by standard methods. Patient 1 received AGV Model FP-7 and patient 2 and patient 5 received AGV Model S-2. The AGV implant was fixed to the globe $9 \mathrm{~mm}$ posterior to the limbus. The tube of the AGV was trimmed and inserted into the anterior chamber through a tract created with a 23-gage needle. The tube was well positioned with $1-2 \mathrm{~mm}$ visible in the anterior chamber. A donor scleral graft was used to secure the AGV implant to the host sclera.

\section{Patient I}

A 49-year-old man with ADNIV was evaluated of recurrent uveitis and elevated IOP. He carried the CAPN5 p.L244P mutation. His uveitis was managed with topical steroids and subtenon's triamcinolone acetate injections for 20 years. On examination, his best-corrected visual acuity (BCVA) was 20/50 OD and 20/1,000 OS. He was functionally monocular.
His eye pressure measured as high as $45 \mathrm{mmHg}$ OD and $25 \mathrm{mmHg}$ OS, and it was refractory to a maximum treatment of dorzolamide-timolol, brimonidine, pilocarpine, and latanoprost. He could not tolerate methazolamide (oral carbonic anhydrase inhibitor). On anterior segment examination, his corneal thickness was $556 \mu \mathrm{m}$ OD and $592 \mu \mathrm{m}$ OS, and there was 2+ flare OU. On right eye gonioscopy, the angle was open with visible scleral spur. The angular approach was $40^{\circ}$, and the iris was flat with $1+$ pigmentation of the trabecular meshwork. In the left eye, both the scleral spur and ciliary body were visible and the angular approach was $35^{\circ}$ with $1+$ pigmentation of the trabecular meshwork. He was pseudophakic OU. The posterior segment exam showed $2+$ vitreous cells OU, vitreous opacities, and a 3+ ERM OD and 1+ ERM OS. He also had significant cystoid macular edema (CME) and inferior thinning of the optic disk OD (Figure 1A). He had significant media opacity, due to intraocular fibrosis and tractional retinal detachment, which obscured view of his optic disk OS (Figure 1B). There was $360^{\circ}$ of diffuse pigmentary clumping OU without any retinal tears or detachment OD. These findings were consistent with early stage III ADNIV OD and stage IV ADNIV OS.

On Goldmann perimetry, the right eye showed diffuse constriction, superior $>$ inferior (Figure 1C). In the left eye, he had extensive visual field losses with only an island of central and inferotemporal visual field remaining (Figure 1D). Stratus optic disk optical coherence tomography (OCT) showed no losses of retinal nerve fiber layer (RNFL) thickness OD, but severe losses were evident OS, especially in the nasal and temporal quadrants (Figure 1E-F).

He elected to undergo FA implantation OD for the management of his uveitis and CME. Given his history of steroid-induced ocular hypertension, it was decided to perform AGV (Model FP7) surgery, concurrent with the FA implantation. During his postoperative course, he did not require antiglaucoma medication OD. His BCVA improved to $20 / 50$ by the second postoperative month, and his IOP remained $<21 \mathrm{mmHg}$ during 1 -year of follow-up.

\section{Patient 2}

At age 24, an ADNIV man with a CAPN5 p.R243L mutation underwent FA implantation OU for control of posterior uveitis, which stabilized his visual acuity at 20/30 OU. Over the next 3 years, however, his vision declined, due to photoreceptor degeneration and intraocular fibrosis, and his IOP steadily increased, even with the combination of dorzolamide-timolol, brimonidine, and latanoprost. He deferred replacement of the FA implants, which exhausted after $\sim 2.5$ years. 

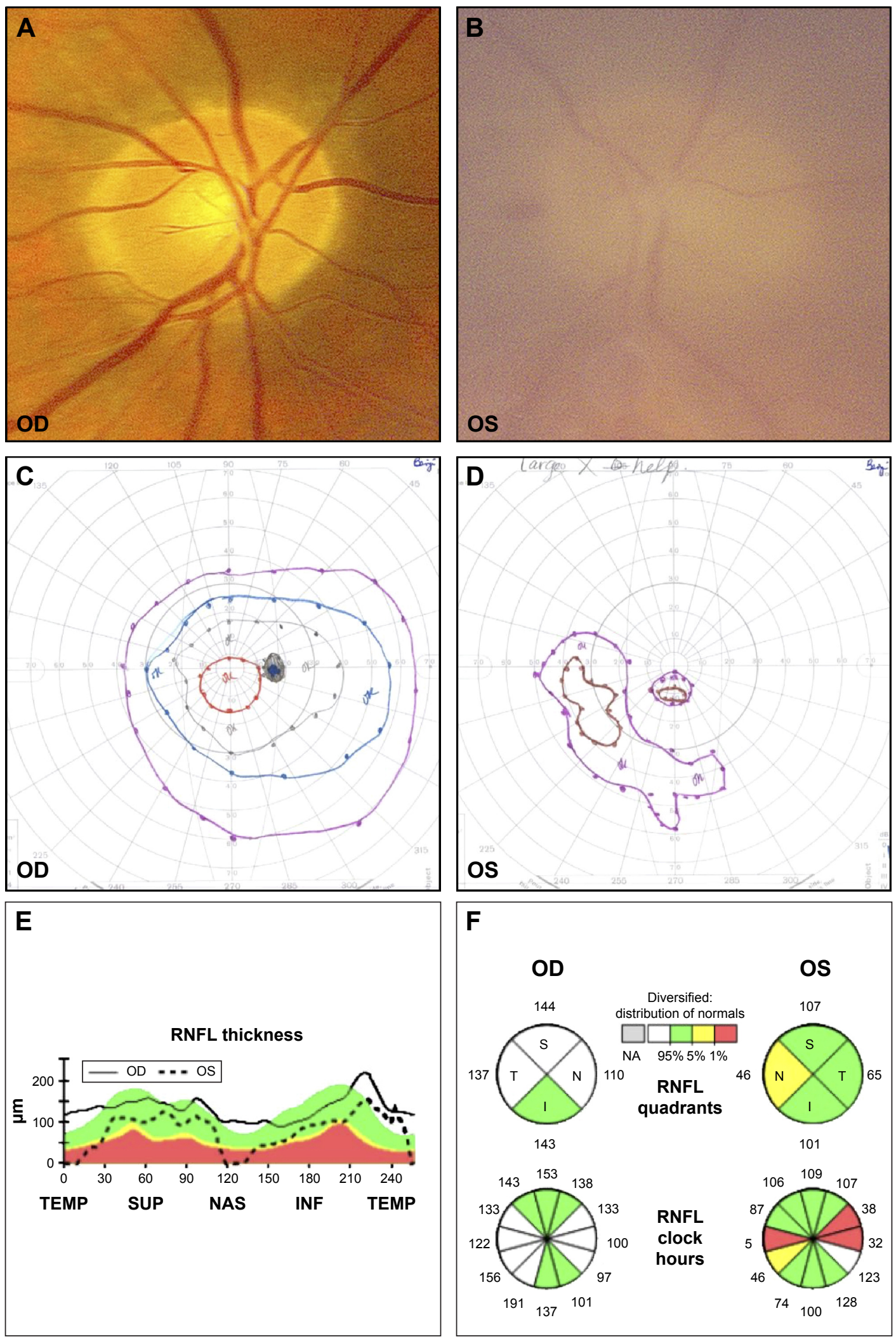

Figure I Patient I.

Notes: (A) OD optic disk image shows vertically oval cup with INF thinning of the NRR. (B) OS optic disk image with media haze and diffuse disk pallor. (C) OD Goldmann perimetry with diffuse constriction, SUP > INF. (D) OS Goldmann perimetry shows remaining island of central and inferotemporal visual field. (E) Stratus OCT RNFL thickness analysis OD shows no RNFL loss. (F) OS RNFL quadrant and analysis show severe losses ( $<1 \%$ of normative data) in the NAS and TEMP quadrants. OS RNFL clock hour analysis corroborates RNFL thickness findings. OD RNFL quadrant and clock hour analyses were both normal.

Abbreviations: INF, inferior; NRR, neuroretinal rim; SUP, superior; OCT, optical coherence tomography; RNFL, retinal nerve fiber layer; NAS, nasal; TEMP, temporal; NA, not applicable; S, superior; N, nasal; T, temporal; I, inferior. 
At age 27, he required AGV surgery OU for IOP control. His BCVA was 20/100 OD and 20/200 OS, and his IOP was $41 \mathrm{mmHg}$ OD and $32 \mathrm{mmHg}$ OS. His corneal thickness was $589 \mu \mathrm{m}$ OD and $597 \mu \mathrm{m}$ OS. On gonioscopy, the ciliary body was visible with the angular approach of $40^{\circ} \mathrm{OU}$. The iris was flat with deep insertion, and there was no pigmentation in the trabecular meshwork. Posterior chamber intraocular lenses were well centered. On posterior examination, he had vitreous membrane attached to the optic nerve head OU and macular traction OS. The optic disk showed evidence of inferior thinning of the neuroretinal rim OD (Figure 2A and B). On Goldmann perimetry, he had significant superior visual field losses OD (Figure 2C) and mild superotemporal visual field constriction OS (Figure 2D).

One year after AGV surgery, his IOP was $25 \mathrm{mmHg}$ OD and $20 \mathrm{mmHg}$ OS. Because of ongoing intraocular fibrosis, common in ADNIV eyes, he suffered from pupillary block with iris bombe and acute angle-closure glaucoma OD. A laser peripheral iridotomy corrected this. With the addition of dorzolamide-timolol, brimonidine, and latanoprost $\mathrm{OU}$, his IOP improved to $19 \mathrm{mmHg}$ OD and $15 \mathrm{mmHg}$ OS by postoperative year 2. At postoperative year 3, his IOP was $17 \mathrm{mmHg}$ OD and $11 \mathrm{mmHg}$ OS. His BCVA OD was 20/100,
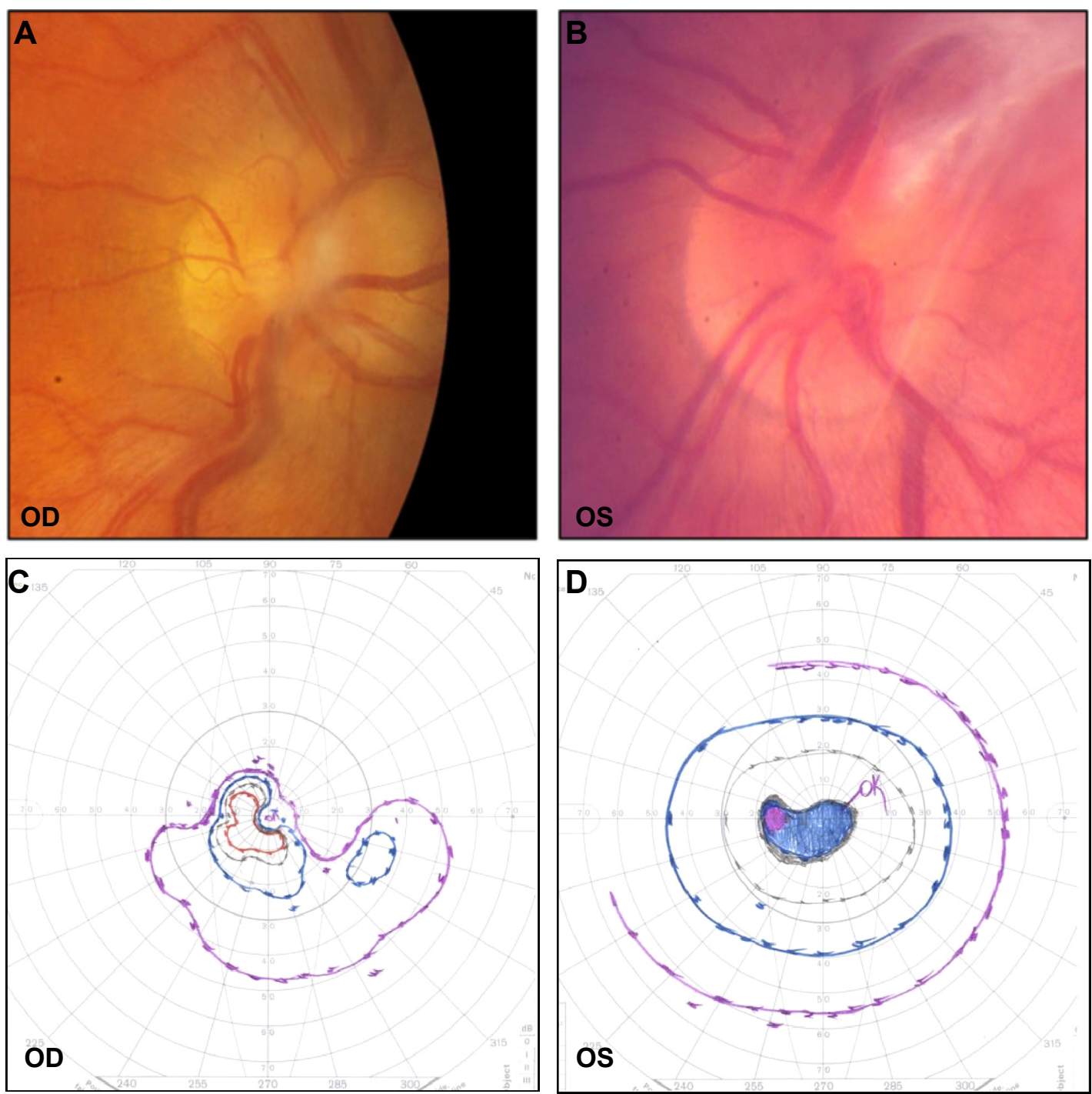

Figure 2 Patient 2.

Notes: (A) OD disk image showing fibrous membrane over disk with inferior thinning of NRR. There is no NVD. (B) OS optic disk appears hyperemic with dense fibrous membrane. There is no NVD. (C) OD Goldmann perimetry shows superior visual field loss. (D) OS Goldmann perimetry shows mild constriction of the superotemporal visual field.

Abbreviations: NRR, neuroretinal rim; NVD, neovascularization of the disk. 
which was equal to his preoperative BCVA, but there was a moderate loss of acuity OS to 20/250.

\section{Patient 3}

A 36-year-old ADNIV woman with a CAPN5 p.R243L mutation was evaluated for chronic CME and uveitis. She was managed with methazolamide for chronic CME, and combination of subtenon's triamcinolone acetate and prednisolone for uveitis. However, she continued to suffer from bouts of recurrent uveitis and underwent FA implantation OU. Her BCVA was 20/40 OD and 20/200 OS. Her IOP was $13 \mathrm{mmHg}$ OD and $14 \mathrm{mmHg}$ OS. Her angles were open, and there was $2+$ flare with rare cells OU. Her posterior segment examination showed $1+$ vitreous cells OU, peripheral retinal pigment epithelium changes OU, mild ERM OU, disk pallor OU (Figure 3A and B), CME OU, attenuated vessels OD (Figure 3A), and early neovascularization of the peripheral retina OS. Goldmann perimetry showed an intact visual field OD and mild superotemporal constriction OS (Figure 3C and D).

To control her uveitis and CME, she underwent FA implantation. After 6 months, her IOP increased to $24 \mathrm{mmHg}$ OD. She was started on timolol OU, and her IOP OD improved to a mean of 16 for the next 2 years. Her IOP OS remained at a mean of 14 . Her BCVA OD remained relatively stable for 2 years, but her left eye acuity began to decline due to intraocular fibrosis and retinal degeneration, eventually becoming prephthisical after 3 years.

She never required glaucoma surgery. Her interval OD Goldmann perimetry after 14 years showed severe constriction with a central scotoma (Figure 3E). Stratus optic disk OCT RNFL thickness showed mild thinning superiorly but was otherwise normal (Figure 3E, F, and G). This suggested that pigmentary degeneration was causing the visual field loss.

\section{Patient 4}

A 66-year-old ADNIV woman with a CAPN5 p.R243L mutation was treated with prednisolone for chronic iritis and required topical brimonidine-timolol for elevated IOP. On examination, her BCVA was 20/50 OD and 20/200 OS and her IOP was $20 \mathrm{mmHg}$ OD and $10 \mathrm{mmHg}$ OS. Her corneal thickness was $602 \mu \mathrm{m}$ OD and $621 \mu \mathrm{m}$ OS. She had 1+ cell and 1+ flare OU and bilateral pseudophakia. On posterior segment examination, she had dense vitreous hemorrhage OS, scattered retinal pigment epithelium clumps OU, markedly attenuated vessels OU, and optic disk pallor OU (Figure 4A and B). Goldmann perimetry showed severe bilateral visual field loss (Figure 4C and D). Stratus OCT RNFL thickness showed marked superior, nasal, and inferior thinning OU (Figure 4E and F).

Her recurrent uveitis, CME, and vitreous hemorrhage OS were refractory to treatment with prednisolone, methotrexate, triamcinolone, and bevacizumab. At age 68, she underwent FA implantation in her left eye. One week postoperatively, her BCVA was 20/300 and her left eye was hypotonous with an IOP of $4 \mathrm{mmHg}$. Her brimonidine-timolol eye drop was withheld OS. By postoperative month 1, her IOP normalized to $18 \mathrm{mmHg}$ OS, and between postoperative months 3-12, her IOP ranged between 7 and $14 \mathrm{mmHg}$. At postoperative month 15, her vision improved to 20/250; however, she had a recurrent hypotony OS with IOP $<4 \mathrm{mmHg}$. Her IOP continued to fluctuate between 4 and $42 \mathrm{mmHg}$, sometimes necessitating topical antiglaucoma medication.

At age 69, she underwent FA implantation OD for chronic posterior uveitis. Preoperatively, her BCVA was 20/30 and her IOP was $13 \mathrm{mmHg}$ OD. However, her IOP increased to $30 \mathrm{mmHg}$ by postoperative week 6 and brimonidine-timolol was resumed. At postoperative month 5 , her IOP increased to $32 \mathrm{mmHg}$ OD and she was started on dorzolamide-timolol. One week later, her IOP dropped to $9 \mathrm{mmHg}$. Her IOP continued to fluctuate between 7 and $33 \mathrm{mmHg}$. The high IOPs were managed with intermittent use of topical antiglaucoma medication, and she has not required $\mathrm{AGV}$ surgery.

\section{Patient 5}

A 26-year-old woman with ADNIV carried a CAPN5 p.K250N mutation. Her bilateral intermediate uveitis was managed with topical steroids, subtenon's triamcinolone acetate injections, intravitreal kenalog, and oral methotrexate for 3 years. However, she continued to have recalcitrant $\mathrm{CME}$ limiting her vision in both eyes. Her BCVA was 20/40 OD and 20/200 OS. IOP was $12 \mathrm{mmHg}$ OU. Her corneal thickness was $584 \mu \mathrm{m}$ OD and $581 \mu \mathrm{m}$ OS. She had 1+ anterior chamber cells OU. Gonioscopy (Spaeth classification) of both eyes revealed scleral spur compressible to ciliary body band, $40^{\circ}$ insertion, a regular and flat configuration of the peripheral iris, and minimal pigmentation of the trabecular meshwork. Peripheral anterior synechiae were noted OS. She had a $2+$ nuclear sclerotic cataract OD and a 1+ nuclear sclerotic cataract/posterior subcapsular cataract OS. The posterior segment examination was significant for $2+$ vitreous cell OU. Optic disks were pink and sharp, with a cup-disk ratio of $0.3 \mathrm{OU}$. Trace ERMs were present OU. The peripheral retinal had 

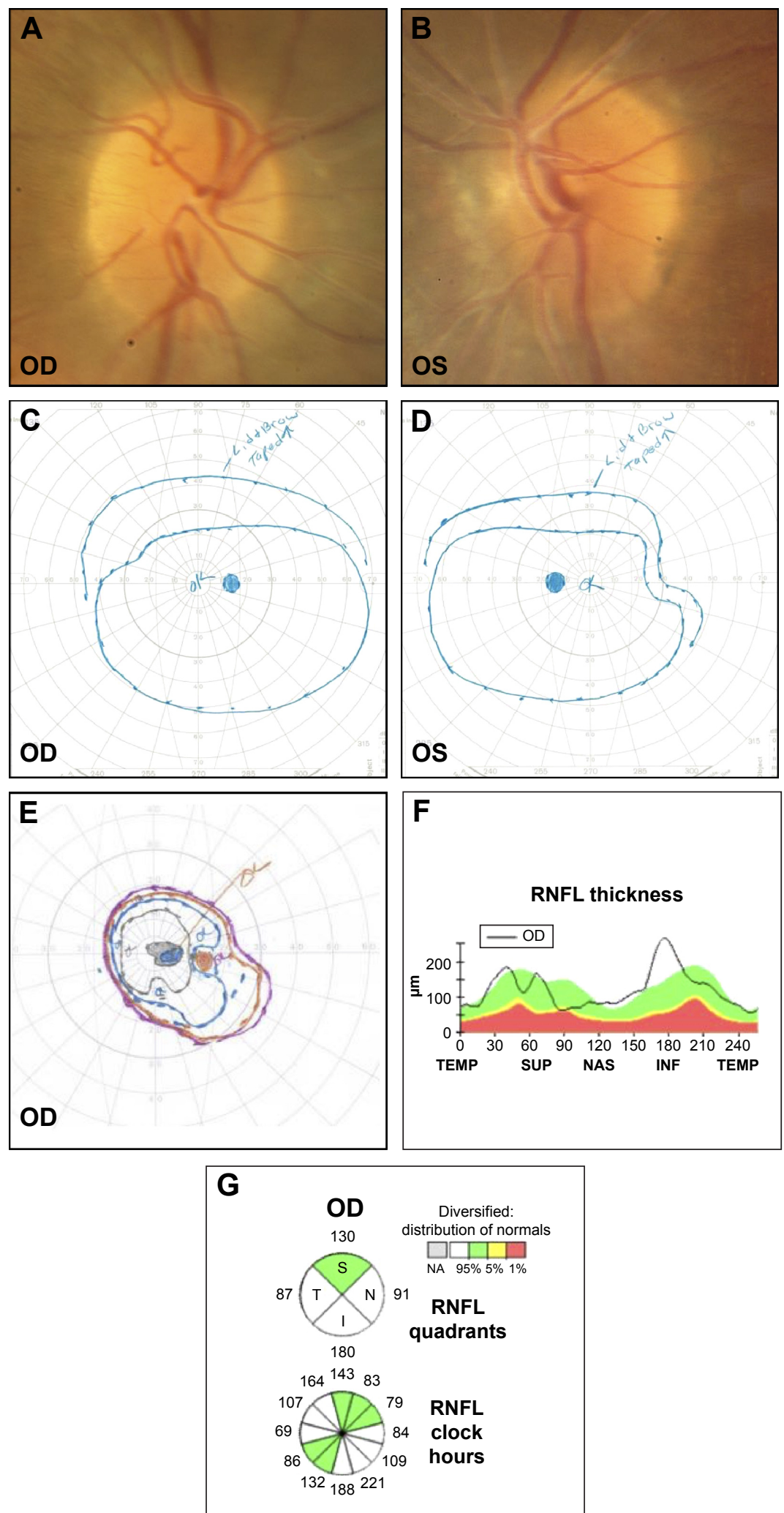

Figure 3 Patient 3.

Notes: (A) OD optic disk has pink and regular NRR without peripapillary atrophy. (B) OS optic disk was similar with fibrous membrane and vessel sheathing. (C) Initial OD Goldmann perimetry with relatively intact visual field. (D) Initial OS Goldman perimetry with superotemporal constriction. (E) Interval OD Goldmann visual field after 14 years shows marked visual constriction with central scotoma (OS unavailable since the patient's left eye became phthisical). (F) OD stratus OCT shows mild RNFL thinning superiorly, otherwise RNFL thickness is normal. (G) Normal RNFL quadrant and RNFL clock hour analyses OD.

Abbreviations: NRR, neuroretinal rim; OCT, optical coherence tomography; RNFL, retinal nerve fiber layer; TEMP, temporal; SUP, superior; NAS, nasal; INF, inferior; NA, not applicable; S, superior; N, nasal; T, temporal; I, inferior. 

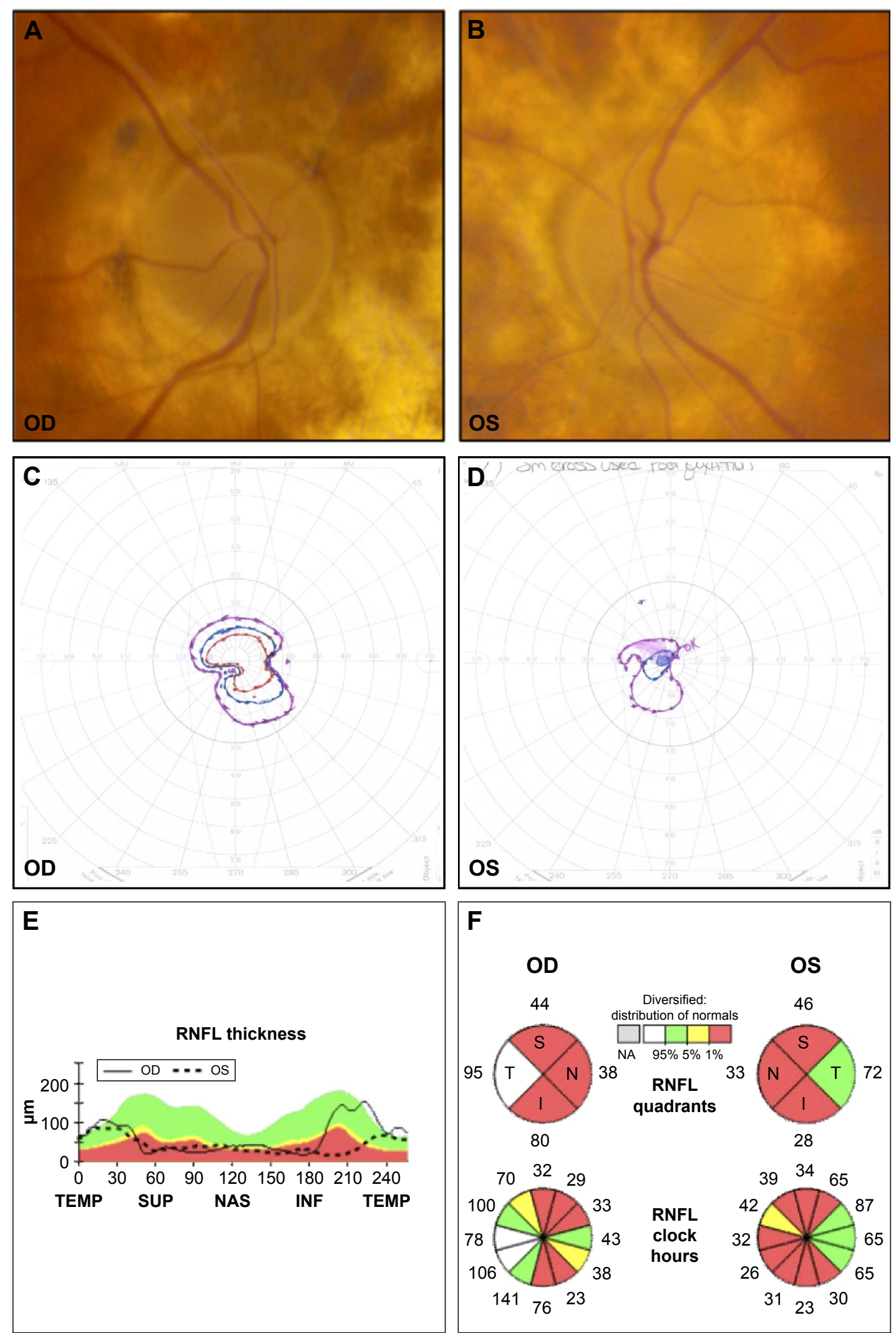

\section{$\mathbf{F}$}

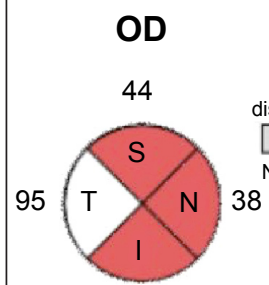

80

70

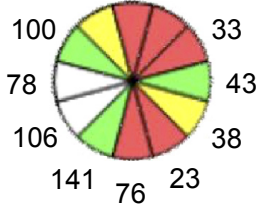

\section{os}

Diversified: distribution of normals NA $95 \% 5 \% 1 \%$

RNFL quadrants

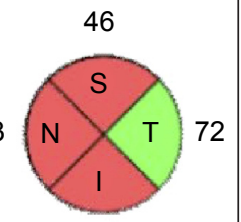

28

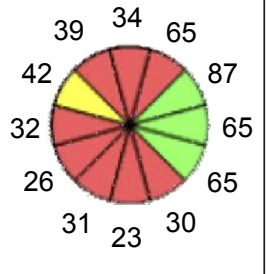

RNFL

clock hours

Figure 4 Patient 4 .

Notes: (A and B). Bilateral disk pallor with loss of disk capillaries and attenuation of major vessels, suggestive of optic atrophy. (C) Goldmann perimetry with severe visual field loss and preservation of central vision OD. (D) Goldmann perimetry with severe visual field loss and split fixation OS. (E) Stratus OCT RNFL thickness analysis OU showed marked SUP, NAS, and INF thinning ( $<1 \%$ of normal). (F) RNFL quadrant and clock hours were consistent with the RNFL thickness findings.

Abbreviations: OCT, optical coherence tomography; RNFL, retinal nerve fiber layer; SUP, superior; NAS, nasal; INF, inferior; TEMP, temporal; NA, not applicable; $\mathrm{S}$, superior; N, nasal; T, temporal; I, inferior. 
diffuse pigmentary clumping OU, without tears or detachments. She had snowballs in the inferior periphery. OCT revealed CME OU. She elected to undergo combined pars plana vitrectomy (PPV)/cataract extraction (CE)/intraocular lens (IOL)/fluocinolone acetonide (FA) implantation OS, with vision improving to $20 / 80$.

Five months after her surgery, her IOP measured $22 \mathrm{mmHg}$ OD and $36 \mathrm{mmHg}$ OS despite treatment with topical brimonidine, dorzolamide-timolol, latanoprost, and oral acetazolamide. Her left optic nerve had a pink neuroretinal rim OS (Figure 5A). Her Humphrey visual field (24-2) indicated inferior and superior arcuate defects OU, OS worse than OD (Figure 5B and C). She elected to undergo implantation of an AGV in her left eye for management of her glaucoma. During her postoperative course, she continued to require antiglaucoma drops in both eyes but was able to discontinue oral acetazolamide.

At the age of 29, she continued to have progression of her cataract and worsening CME OD. She underwent PPV/CE IOL/FA implantation in the right eye. IOP in the right eye measured $35 \mathrm{mmHg} 1$ month after her PPV despite maximum topical therapy. An AGV was implanted in the right eye. She was maintained on topical antiglaucoma medication with stable central acuity and glaucomatous findings noted on her visual fields for 3 years.

Despite continued subtenon's steroid injections OU, she continued to have CME in both eyes and underwent another PPV with a second FA implantation OU. She developed band keratopathy in both eyes, OD worse than OS. She underwent ethylene diamine tetraacetic acid chelation OD with improvement in her vision and symptoms.

Five years after her last AGV surgery, her IOP was $5 \mathrm{mmHg}$ OD and $9 \mathrm{mmHg}$ OS. BCVA was 20/40 OD and 20/100 OS. Interval Humphrey visual field indicated progression of glaucomatous changes OU (Figure 5D and E).

\section{Discussion}

Uveitis is the fifth leading cause of vision loss in the developed world. It is often a chronic disease, and long-term steroid therapy increases likelihood of steroid-induced complications such as cataracts, infections, and glaucoma. About one-third of all eyes experience IOP elevation with steroid treatment, and inflammatory uveitis compounds this risk by diminishing trabecular outflow., 5 Although uveitis can also cause aqueous hyposecretion, due to inflammation of the ciliary body, this IOP-lowering effect is usually overpowered by the increased resistance to trabecular drainage from inflammatory infiltrates and endothelial dysfunction. On balance, these processes often result in increased IOP and predispose patients to glaucoma.
In the setting of inflammatory uveitis, glaucoma can be secondary to steroid therapy, inflammation, or angle neovascularization. ADNIV patients are at risk of developing secondary glaucoma via all the three mechanisms, and they also develop intraocular fibrosis. Despite these significant risk factors, some ADNIV patients do not develop elevated IOP or glaucoma. In this series, patient 3 had normal appearing optic disks without evidence of glaucomatous change, despite $>40$ years of ADNIV-related pathology. Furthermore, her eye pressures were well controlled for up to 3 years following FA implantation. This suggests that a subset of patients may not develop complications of glaucoma, despite decades of ADNIV and steroid therapy. Furthermore, the exact genetic defect does not seem to make a difference, since glaucoma developed in all the three CAPN5 mutations varied between patients with the same genetic defect and between eyes. ${ }^{3,10,12}$ This supports the concept that there is significant phenotypic variance in ADNIV features. ${ }^{12}$

ADNIV uveitis generally fails management with topical steroids and steroid-sparing therapy. Oral prednisone at $>10 \mathrm{mg} / \mathrm{d}$ usually causes too severe side effects to be used long term; therefore, ADNIV patients receive FA implants. Friedman et $\mathrm{al}^{13}$ reported that FA implant recipients were two to three times more likely to develop elevated IOP and require IOP-lowering therapy compared to patients receiving systemic steroid therapy. ${ }^{13}$ Furthermore, Bollinger et al ${ }^{14}$ also reported that $75 \%$ of patients with FA implants required IOP-lowering drops and almost half had IOP $>30 \mathrm{mmHg}$ within the first postoperative year. Additionally, $45 \%$ of patients receiving FA implants required glaucoma surgery by 36 months following FA implantation. ${ }^{15}$ Given the risks of elevated IOP and glaucoma associated with FA implantation and the baseline risk conferred by ADNIV itself, it is encouraging that some ADNIV patients - such as patient 3- might not develop glaucoma or steroid-induced ocular hypertension.

Neovascular glaucoma was previously a major complicating factor in ADNIV patients, but we reported that FA implantation seems to have largely resolved this. ${ }^{10}$ In one instance, prior to FA implantation, ADNIV optic disk neovascularization had resolved following a course of oral prednisone and methotrexate. This is consistent with the findings of Sanislo et al ${ }^{16}$ regarding treatment of optic disk neovascularization with oral and periocular corticosteroids, and the FA implants are likely to have had a similar effect. ${ }^{10}$ The FA implants are also effective at controlling ADNIV uveitis; ${ }^{15}$ however, steroid-induced ocular hypertension is an emerging concern. This conundrum makes combined FA-AGV surgery particularly attractive. FA implantation and 

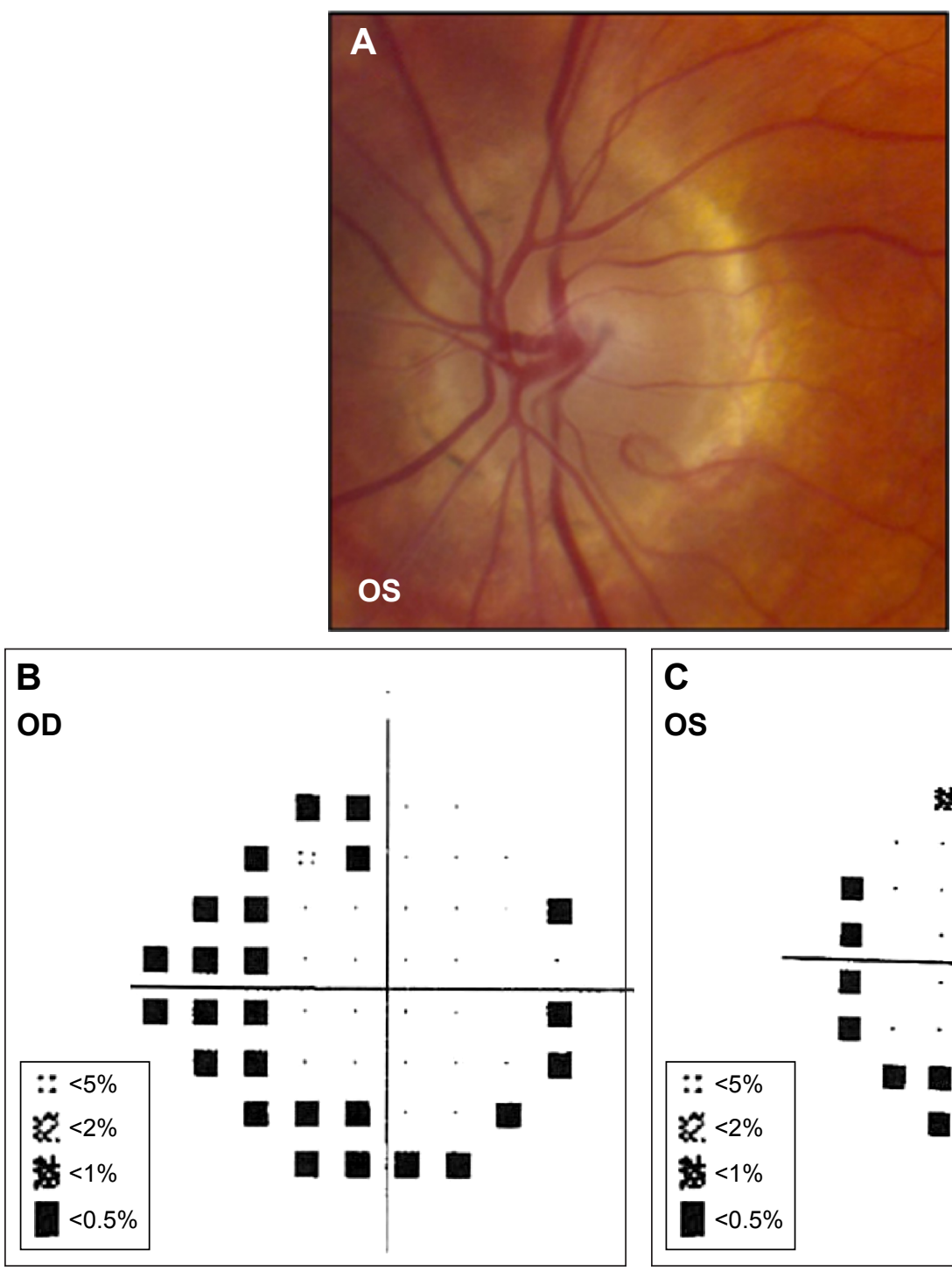

C
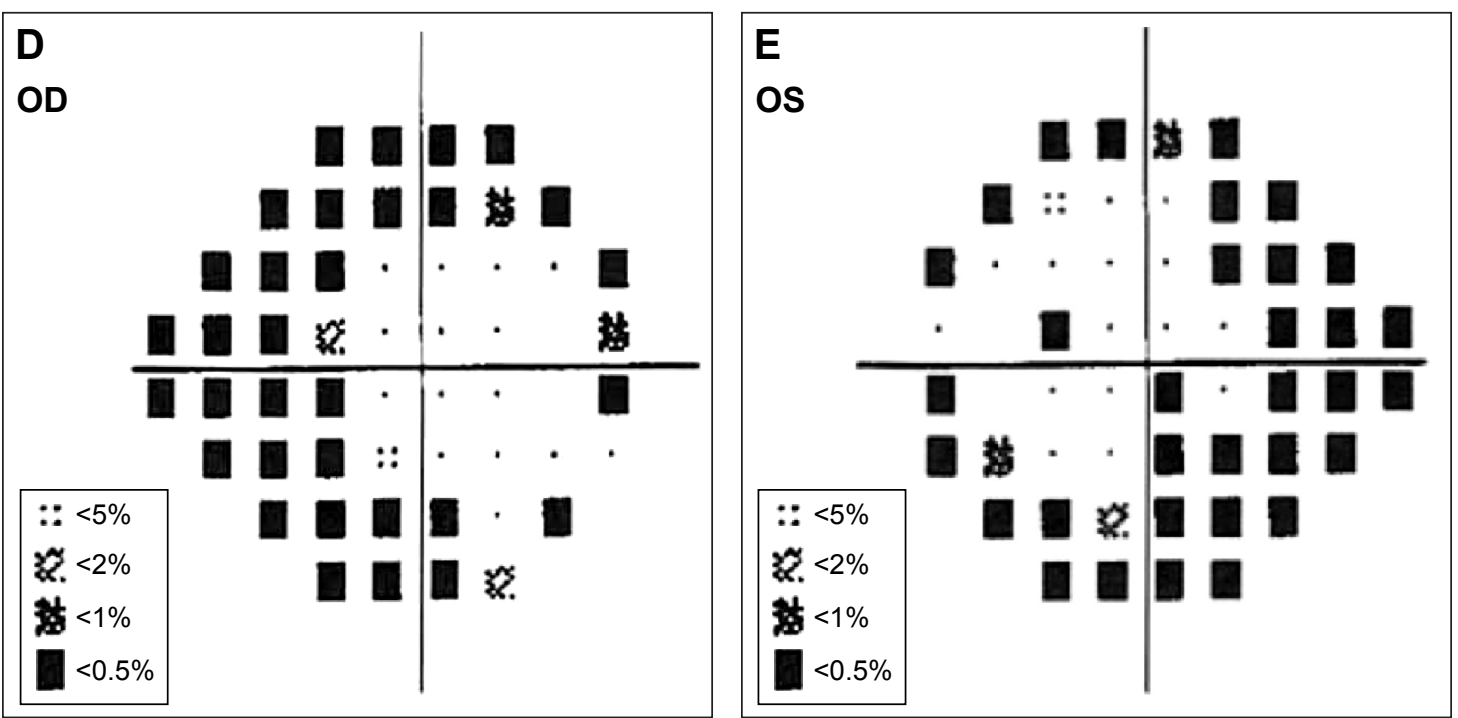

Figure 5 Patient 5.

Notes: (A) OS optic disk has pink and regular NRR (OD examination was similar to OS examination). (B and C) Humphrey Visual Field 24-2 SITA-Standard protocol with superior and inferior arcuate defects OS > OD, and superior and inferior nasal step defects OU. (D and E) Interval Humphrey Visual Field 24-2 SITA-Standard protocol (obtained 6 years later) demonstrates glaucomatous progression with denser superior arcuate defects OU.

Abbreviation: NRR, neuroretinal rim. 
glaucoma valve surgery have been successfully performed in a single surgical session. ${ }^{8}$ Nonetheless, it is often difficult to determine in advance which ADNIV patients are likely to be steroid responders following FA implantation, and hence, good candidates for combined AGV-FA surgery.

IOP fluctuation is challenging to manage in glaucoma patients. In our series, patient 4's IOPs became very labile following FA implantation. The possible causes of hypotony include FA surgery, uveitis, and FA-associated hypotony. Bollinger et $\mathrm{al}^{14}$ reported that, following FA surgery, 35\% of patients developed hypotony $(<5 \mathrm{mmHg})$. For this reason, some glaucoma surgeons avoid a large plate Baerveldt tube shunt (eg, Baerveldt 350) with no valve, which can lead to postoperative hypotony.

Three multicenter clinical trials have demonstrated that FA implants are very effective at decreasing uveitis recurrence. ${ }^{15}$ However, steroid-induced IOP elevation, as described above, is a common complication of FA implantation. ${ }^{14} \mathrm{AGV}$ implant is effective in treating uveitic glaucoma with few surgical complications. ${ }^{17}$ Elevated IOP in the early postoperative period may be due to AGV obstruction by fibrin, blood, iris, vitreous membranes, or silicone oil. In one study, this was observed in $11 \%$ of eyes following AGV implantation. The most common causes of blockage were iris tissues and fibrinous membranes followed by neovascular membrane and iridocorneal endothelial membrane. ${ }^{18}$ ADNIV patients can have an exuberant inflammatory response following intraocular surgery. ${ }^{7}$ Following AGV surgery, patient 2 still required topical therapy for IOP control. It is possible that his AGV implant become less functional due to fibrosis around the tube plate. ${ }^{7}$ Successful AGV surgery combined with adjunctive mitomycin $\mathrm{C}$ and fluorouracil has been reported by Alvarado et al. ${ }^{19}$ However, others have found no benefit of using adjunctive antifibrotic agents with tube shunts. ${ }^{20}$ At this point, we do not recommend adjunctive agents in ADNIV patients.

\section{Conclusion}

ADNIV is a complex hereditary autoimmune uveitis where disease management poses several challenges. In the past, severe progressive vision loss in ADNIV due to intraocular inflammation, neovascular glaucoma, and tractional retinal detachment made optic nerve monitoring less important, since these end-stage eyes rapidly became blind. The recent use of long-term steroid implants has delayed disease progression and controlled other aspects of ADNIV. This has made monitoring of glaucoma more important. The complex pathology of ADNIV and superimposed retinal degeneration makes interpretation of glaucoma studies challenging, and the use of OCT imaging in ADNIV may be more reliable than perimetry data and clinical examination. FA implants effectively control uveitis in ADNIV patients; however, they predispose patients to complications such as cataracts and steroid-response glaucoma. ADNIV patients with elevated IOP refractory to medical management were treated with AGV surgery. The findings from this series suggest that some ADNIV patients have good outcomes with combined FA-AGV surgery.

\section{Acknowledgments}

VBM was supported by the National Institutes of Health's grants K08EY020530 and R01EY016822, the Doris Duke Charitable Foundation's grant 2013103, and Research to Prevent Blindness, New York, NY, USA.

\section{Disclosure}

The authors report no conflicts of interest in this work.

\section{References}

1. Bennett SR, Folk JC, Kimura AE, Russell SR, Stone EM, Raphtis EM. Autosomal dominant neovascular inflammatory vitreoretinopathy. Ophthalmology. 1990;97(9):1125-1135. [discussion 1126-1135].

2. Mahajan VB, Skeie JM, Bassuk AG, et al. Calpain-5 mutations cause autoimmune uveitis, retinal neovascularization, and photoreceptor degeneration. PLoS Genet. 2012;8(10):e1003001.

3. Bassuk AG, Yeh S, Wu S, et al. Structural modeling of a novel CAPN5 mutation that causes uveitis and neovascular retinal detachment. PLoS One. 2015;10(4): 0122352.

4. Wert KJ, Bassuk AG, Wu WH, et al. CAPN5 mutation in hereditary uveitis: the R243L mutation increases calpain catalytic activity and triggers intraocular inflammation in a mouse model. Hum Mol Genet. 2015;24(16):4584-4598.

5. Siddique SS, Suelves AM, Baheti U, Foster CS. Glaucoma and uveitis. Surv Ophthalmol. 2013;58(1):1-10.

6. Sung VC, Barton K. Management of inflammatory glaucomas. Curr Opin Ophthalmol. 2004;15(2):136-140.

7. Tlucek PS, Folk JC, Sobol WM, Mahajan VB. Surgical management of fibrotic encapsulation of the fluocinolone acetonide implant in CAPN5associated proliferative vitreoretinopathy. Clin Ophthalmol. 2013;7: 1093-1098.

8. Malone PE, Herndon LW, Muir KW, Jaffe GJ. Combined fluocinolone acetonide intravitreal insertion and glaucoma drainage device placement for chronic uveitis and glaucoma. Am J Ophthalmol. 2010;149(5): 800-806.e1.

9. Ahmad ZM, Hughes BA, Abrams GW, Mahmoud TH. Combined posterior chamber intraocular lens, vitrectomy, Retisert, and pars plana tube in noninfectious uveitis. Arch Ophthalmol. 2012;130(7):908-913.

10. Tlucek PS, Folk JC, Orien JA, Stone EM, Mahajan VB. Inhibition of neovascularization but not fibrosis with the fluocinolone acetonide implant in autosomal dominant neovascular inflammatory vitreoretinopathy. Arch Ophthalmol. 2012;130(11):1395-1401.

11. Kocabora MS, Yilmazli C, Taskapili M, Gulkilik G, Durmaz S. Development of ocular hypertension and persistent glaucoma after intravitreal injection of triamcinolone. Clin Ophthalmol. 2008;2(1):167-171.

12. Rowell HA, Bassuk AG, Mahajan VB. Monozygotic twins with CAPN5 autosomal dominant neovascular inflammatory vitreoretinopathy. Clin Ophthalmol. 2012;6:2037-2044. 
13. Friedman DS, Holbrook JT, Ansari H, et al. Risk of elevated intraocular pressure and glaucoma in patients with uveitis: results of the multicenter uveitis steroid treatment trial. Ophthalmology. 2013;120(8):1571-1579.

14. Bollinger KE, Smith SD. Prevalence and management of elevated intraocular pressure after placement of an intravitreal sustained-release steroid implant. Curr Opin Ophthalmol. 2009;20(2):99-103.

15. Bollinger K, Kim J, Lowder CY, Kaiser PK, Smith SD. Intraocular pressure outcome of patients with fluocinolone acetonide intravitreal implant for noninfectious uveitis. Ophthalmology. 2011;118(10):1927-1931.

16. Sanislo SR, Lowder CY, Kaiser PK, et al. Corticosteroid therapy for optic disc neovascularization secondary to chronic uveitis. Am J Ophthalmol. 2000;130(6):724-731.
17. Da Mata A, Burk SE, Netland PA, Baltatzis S, Christen W, Foster CS. Management of uveitic glaucoma with Ahmed glaucoma valve implantation. Ophthalmology. 1999;106(11):2168-2172.

18. Huang MC, Netland PA, Coleman AL, Siegner SW, Moster MR, Hill RA. Intermediate-term clinical experience with the Ahmed glaucoma valve implant. Am J Ophthalmol. 1999;127(1):27-33.

19. Alvarado JA, Hollander DA, Juster RP, Lee LC. Ahmed valve implantation with adjunctive mitomycin $\mathrm{C}$ and 5-fluorouracil: long-term outcomes. Am J Ophthalmol. 2008;146(2):276-284.

20. Minckler DS, Francis BA, Hodapp EA, et al. Aqueous shunts in glaucoma: a report by the American Academy of Ophthalmology. Ophthalmology. 2008;115(6):1089-1098.
Clinical Ophthalmology

\section{Publish your work in this journal}

Clinical Ophthalmology is an international, peer-reviewed journal covering all subspecialties within ophthalmology. Key topics include: Optometry; Visual science; Pharmacology and drug therapy in eye diseases; Basic Sciences; Primary and Secondary eye care; Patien Safety and Quality of Care Improvements. This journal is indexed on

\section{Dovepress}

PubMed Central and CAS, and is the official journal of The Society of Clinical Ophthalmology (SCO). The manuscript management system is completely online and includes a very quick and fair peer-review system, which is all easy to use. Visit http://www.dovepress.com/ testimonials.php to read real quotes from published authors. 